\title{
Long-term results of oxybutynin use in treating facial hyperhidrosis*
}

\author{
Nelson Wolosker ${ }^{1,2}$ \\ Mariana Krutman ${ }^{1}$ \\ Paulo Kauffman ${ }^{1}$ \\ Pedro Puech-Leão ${ }^{2}$
}

\author{
Marcelo Passos Teivelis ${ }^{1}$ \\ Taiz Pereira Dozono de Almeida Campbell ${ }^{2}$ \\ José Ribas de Campos ${ }^{1,2}$
}

DOI: http://dx.doi.org/10.1590/abd1806-4841.20143272

\begin{abstract}
BACKGROUND: Facial hyperhidrosis can lead to serious emotional distress. Video-assisted thoracic sympathectomy resolves symptoms effectively, though it may be associated with compensatory hyperhidrosis, which may be more common in patients undergoing resection of the second thoracic ganglion. Oxybutynin has been used as a pharmacological approach to facial hyperhidrosis but the long-term results of this treatment are unclear. OвJECTIVE: To evaluate the use of low oxybutynin doses in facial hyperhidrosis patients for at least six months. METHODS: 61 patients were monitored for over six months and assessed according to the following variables: impact of hyperhidrosis on quality of life (QOL) before treatment and after six weeks, evolution of facial hyperhidrosis after six weeks and at the last consultation, complaints of dry mouth after six weeks and on last return visit, and improvement at other hyperhidrosis sites.

REsults: Patients were monitored for 6 to 61 months (median=17 months). Thirty-six (59\%) were female. Age ranged from 17-74 (median:45). Pre-treatment QOL was poor/very poor in $96.72 \%$. After six weeks, 100\% of patients improved QOL. Comparing results after six weeks and on the last visit, $91.8 \%$ of patients maintained the same category of improvement in facial hyperhidrosis, 3.3\% worsened and $4.9 \%$ improved. Dry mouth complaints were common but not consistent throughout treatment. More than $90 \%$ of patients presented moderate/great improvement at other hyperhidrosis sites.

CONCLUSION: Patients who had a good initial response to treatment maintained a good response long-term, did not display tachiphylaxis and experienced improvement on other hyperhidrosis sites.
\end{abstract}

Keywords: Cholinergic antagonists; Hyperhidrosis; Quality of life; Sweating

\section{INTRODUCTION}

Hyperhidrosis is a disease that affects patients'quality of life (QOL), compromising their professional, emotional, social, and leisure activities. ${ }^{1}$ Facial hyperhidrosis (FH) usually starts in adulthood and is less common than the axillary and palmar forms of the disease. ${ }^{1-3}$

Video-assisted thoracic sympathectomy (VATS) has high success rates and low operative risks for the definite treatment of hyperhidrosis, but compensatory hyperhidrosis is usually a troublesome complaint, particularly with facial hyperhidrosis, in which ganglion the resection level is higher (T2). ${ }^{4-7}$

Oxybutynin use has been reported in cases of axillary and palmar hyperhidrosis, including a placebo-controlled trial; short-term results for facial hyper- hidrosis have also been published. , $^{3,-11}$ However, the long-term results of treatment with this anticholinergic drug are unclear.

The aim of this study was to assess the longterm clinical efficacy of a large series of consecutive patients $(n=61)$ with facial hyperhidrosis $(\mathrm{FH})$, treated with low doses of oxybutynin for at least six months (median=17 months).

\section{CASUISTICS}

This was a non-randomized and uncontrolled study, performed in accordance with the standards set out by the relevant Ethics Committees for Analysis of Research Projects on Human Experimentation. Data were retrieved from our dedicated and prospective,

Received on 17.11.2013.

Approved by the Advisory Board and accepted for publication on 11.02.2014

Work performed at the Hospital Israelita Albert Einstein (HIAE) and Hospital das Clínicas da Faculdade de Medicina da Universidade de São Paulo (HCFMUSP) - São Paulo (SP), Brazil.

Financial Support: None.

Conflict of Interest: None.

Hospital Israelita Albert Einstein (HIAE) - São Paulo (SP), Brazil.

Hospital das Clínicas da Faculdade de Medicina da Universidade de São Paulo(HC-FMUSP) - São Paulo (SP), Brazil. 
standardized institutional protocol, which has been in use at our departments since 2001 for all hyperhidrosis patients.

From September 2007 to September 2013, a total of 98 consecutive patients, whose main complaint was facial hyperhidrosis, were enrolled at our institutions. Seven patients failed to attend their first return visits. Of the remaining 91, twelve (13.2\%) patients did not improve after six weeks and left the study due to their nonimprovement following pharmacological treatment. A further two additional patients reported severe dry mouth and left the study. Seventy-seven patients were treated with oxybutynin for six weeks. Sixteen were monitored for under six months, while 61 were monitored for over six months. The latter were the patients analyzed. Figure 1 graphically represents the series.

There were 36 females and 25 males, ranging in age from 17 to 74 years (median: 45 years). This subgroup was comparable to the general group of 98 patients, in terms of age, gender, distribution and complaints. in table 1.

The patients' demographics are displayed

FH was not their only complaint as all of them presented hyperhidrosis at other body sites: thoracic and abdominal hyperhidrosis in 37 patients $(60.7 \%)$, axillary hyperhidrosis in 27 patients $(44.3 \%)$, palmar hyperhidrosis in 13 (21.31\%), and plantar hyperhidrosis in $7(11.48 \%)$.

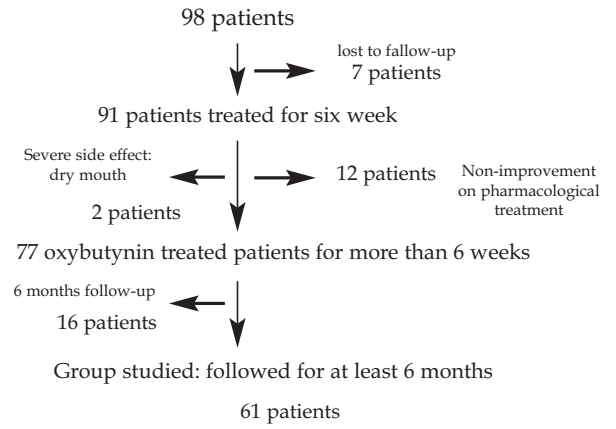

FIGURE 1: Follow-up algorithm of pharmacologically treated patients with facial hyperhidrosis

TABLE 1: Demographics

\begin{tabular}{|c|c|}
\hline Male & $25(41.0 \%)$ \\
\hline Female & $36(59.0 \%)$ \\
\hline Age (range) & $17-74$ \\
\hline Age (average \pm Standard Deviation) & $43.9 \pm 13.9$ \\
\hline Age (median) & 45.0 \\
\hline $\begin{array}{l}\text { Body Mass Index (BMI) - } \\
\text { Average } \pm \text { Averages Index (BMI) }\end{array}$ & $29.86 \pm 4.5$ \\
\hline BMI (Median) & 29.27 \\
\hline BMI (range) & $19.33-42.59$ \\
\hline
\end{tabular}

All patients followed the same protocol: during the first week they received $2.5 \mathrm{mg}$ of oxybutynin once a day at bedtime. From day 8 to 21 , they received $2.5 \mathrm{mg}$ twice a day, and from day 22 onwards, they received $5 \mathrm{mg}$ twice a day.

Three evaluations were made for the purpose of this study. The first occurred before pharmacological treatment, the second following 6 weeks of medical treatment, and the last after at least 24 weeks subsequent to protocol initiation. The following were evaluated: initial quality of life - using a validated questionnaire for hyperhidrotic patients, clinical improvement of facial hyperhidrosis after 6 weeks, quality of life following six weeks of treatment, clinical improvement of facial hyperhidrosis at the last visit (that occurred at least six months after treatment), complaint of pharmacological side effects after six weeks and at the last visit, and the patient's impressions of improvement at other hyperhidrosis sites during the last visit. . $^{1-6,12,13}$

Patients completed the clinical improvement questionnaire according to their subjective perception of improvement of sudoresis. They evaluated it on a scale ranging from 0 to 10 , such that 0 represented no improvement, while 10 represented absence of hyperhidrosis, based on their own assessments, without any intervention or advice from the interviewer. Improvement was graded as follows: null (0); slight (1-4); moderate (5-7); and great (8-10).

The negative impact of hyperhidrosis on QOL before treatment was classified into five different levels, calculated as the summed total score from the protocol (ranging from 20 to 100). The higher the level, the greater the impact and the poorer the QOL, which was scored as follows: very poor (>83), poor (68-83), good (52-67), very good (36-51), and excellent (20-35).

Improvement of QOL after treatment was also classified into the five following different evolution levels: much worse (>83), slightly worse (68-83), equal (52-67), slightly better (36-51), and much better (20-35).

The following parameters were studied: negative impact of hyperhidrosis on QOL before treatment; improvement in QOL after six weeks of treatment; evolution of $\mathrm{FH}$ (comparing results after six weeks of treatment with results at the last follow-up visit) and improvement at the last consultation on the other hyperhidrosis sites and complications.

\section{Statistical analysis}

Means and Standard Deviations (SDs) were used in the descriptive analysis of parametrically distributed continuous variables, whereas frequencies were used for categorical variables. The McNemar test was used to compare self-reported improvement of hyperhidrosis and dry mouth over time, at the two 
consecutive analysis time points, while the kappa coefficient was used for analysis.

The significance level considered for all tests was $\mathrm{P}=0.05$.

\section{RESULTS}

Patients were monitored for at least six months, with periods ranging from 6 to 61 months (median=17 months). Quality of life before treatment is presented in table 2.

Almost $97 \%$ of patients reported poor or very poor quality of life before treatment.

Improvement in Quality of life after six weeks of treatment is presented in table 3 .

All patients had an improved quality of life after 6 weeks.

Improvements in sweating after six weeks and at the last visit are presented in table 4 .

TABLE 2: Pre-treatment Quality of life

\begin{tabular}{lll}
\hline & $\%$ & $\mathbf{N}$ \\
\cline { 2 - 3 } Very poor & 47.54 & 29 \\
Poor & 49.18 & 30 \\
Good & 1.64 & 1 \\
Very good & 1.64 & 1 \\
Excellent & 0 & 0 \\
\hline Total & 100 & 61 \\
\hline
\end{tabular}

TABLE 3: Quality of life after six weeks of treatment

\begin{tabular}{lll}
\hline & $\%$ & $\mathbf{N}$ \\
\cline { 2 - 3 } Much worse & 0 & 0 \\
Slightly worse & 0 & 0 \\
The same & 0 & 0 \\
Slightly better & 60.66 & 37 \\
Much better & 39.34 & 24 \\
\hline Total & 100 & 61 \\
\hline
\end{tabular}

After six weeks of pharmacological treatment, almost $97 \%$ of the patients reported moderate or great improvement in self-perceived sweating. At the last visit, almost $94 \%$ maintained moderate or great improvement in self-perceived sweating.

Comparing the improvement category at the six-week analysis point and last evaluation, $91.8 \%$ of patients remained in the same improvement category, only $3.3 \%$ "downgraded", while $4.9 \%$ improved longterm (Table 4).

Improvement at other hyperhidrosis sites - as reported during the last evaluation - are shown in table $5: 90 \%$ of patients presented moderate or great improvement in the other areas of excessive sweating.

Comparisons between dry mouth complaints after six weeks and at the final visit are shown in table 6. No serious side effects (e.g., constipation, intestinal obstruction, urinary retention) occurred in this population.

\section{DISCUSSION}

Regarding our patients' baseline characteristics, the majority were female (as is customary in hyperhidrosis patients) and older (median: 45 years old) than most patients with other hyperhidrosis sites, who usually seek medical attention in the second or third decade of life. ${ }^{14-16}$

Patients with hyperhidrosis experience a significant decrease in their QOL. Surgical treatment is a good

TABLE 5: Reports of improvement on other sites of hyperhidrosis on the last visit (last visit ranging from 6 to 61 months of treatment - median: 17 months)

\begin{tabular}{|c|c|c|c|}
\hline & Null/Slight & Moderate & Great \\
\hline $\begin{array}{l}\text { Thoraco-abdominal } \\
(\mathrm{n}=37)\end{array}$ & $2(5.4 \%)$ & $14(37.8 \%)$ & $21(56.8 \%)$ \\
\hline Axilar (n=27) & $1(3.7 \%)$ & $11(40.7 \%)$ & $15(55.6 \%)$ \\
\hline Palmar $(n=13)$ & $1(7.7 \%)$ & $4(30.8 \%)$ & $8(61.5 \%)$ \\
\hline Plantar $(n=7)$ & 0 & 0 & $7(100 \%)$ \\
\hline
\end{tabular}

TABLE 4: Comparison of self-assessment of improvement of hyperhidrosis of six weeks of treatment and last visit

\begin{tabular}{|c|c|c|c|c|c|c|}
\hline \multirow{10}{*}{ Improvement after six weeks } & & & \multicolumn{3}{|c|}{$\begin{array}{l}\text { Improvement at last visit (ranging } \\
\text { from } 6 \text { to } 61 \text { months of treatment } \\
\text { - median: } 17 \text { months) }\end{array}$} & \multirow[b]{2}{*}{ Total } \\
\hline & & & Slight & Moderate & Great & \\
\hline & \multirow[t]{2}{*}{ Slight } & Count & 2 & 0 & 0 & 2 \\
\hline & & $\%$ of Total & $3.3 \%$ & $0 \%$ & $0 \%$ & $3.3 \%$ \\
\hline & \multirow[t]{2}{*}{ Moderate } & Count & 2 & 18 & 3 & 23 \\
\hline & & $\%$ of Total & $3.3 \%$ & $29.5 \%$ & $4.9 \%$ & $37.7 \%$ \\
\hline & \multirow[t]{2}{*}{ Great } & Count & 0 & 0 & 36 & 36 \\
\hline & & $\%$ of Total & $0 \%$ & $0 \%$ & $59.0 \%$ & $59.0 \%$ \\
\hline & \multirow{2}{*}{ Total } & Count & 4 & 18 & 39 & 61 \\
\hline & & $\%$ of Total & $6.6 \%$ & $29.5 \%$ & $63.9 \%$ & $100.0 \%$ \\
\hline
\end{tabular}

McNemar test $\mathrm{p}=0.082$. Kappa: 0.839 . Consistency: $91.8 \%$. Worse: $3.3 \%$. Better: $4.9 \%$ 
TABLE 6: Complaint of dry-mouth after six weeks of treatment and on the last visit

\begin{tabular}{|c|c|c|c|c|c|c|c|}
\hline \multirow[t]{3}{*}{ Six weeks } & \multicolumn{4}{|c|}{$\begin{array}{l}\text { Last visit (ranging from } 6 \text { to } 61 \text { months of } \\
\text { treatment - median: } 17 \text { months) }\end{array}$} & \multirow{2}{*}{\multicolumn{2}{|c|}{ Total }} & \multirow[t]{3}{*}{ McNemar $p$} \\
\hline & \multicolumn{2}{|c|}{ Absent/Light } & \multicolumn{2}{|c|}{ Moderate/Severe } & & & \\
\hline & $\mathbf{n}$ & $\%$ & $\mathbf{n}$ & $\%$ & $\mathbf{n}$ & $\%$ & \\
\hline Absent/Light & 14 & $23.0 \%$ & 16 & $26.2 \%$ & 30 & $49.2 \%$ & 0.736 \\
\hline Moderate/Severe & 19 & $31.1 \%$ & 12 & $19.7 \%$ & 31 & $50.8 \%$ & \\
\hline Total & 33 & $54.1 \%$ & 28 & $45.9 \%$ & 61 & $100.0 \%$ & \\
\hline
\end{tabular}

McNemar test $\mathrm{p}=0.736$. Kappa: -0.146. Consistency: $42.7 \%$. Worse: $26.2 \%$. Better: $31.1 \%$

option, but it is related to a frequent and bothersome side effect, namely compensatory hyperhidrosis - which can be more frequent at higher levels of sympathectomy, such as those performed for facial hyperhidrosis, and may be more common among overweight/obese patients. ${ }^{7,17}$ Since the Body Mass Index (BMI) in this series was high (median: $29.27 \mathrm{~kg} / \mathrm{m}^{2}$ ), a significant proportion of our patients would not have been good surgical candidates. Hence, they could benefit from non-surgical treatment for facial hyperhidrosis. Previous studies corroborate the theory that overweightness and obesity do not lower oxybutynin treatment efficiency in the pharmacological treatment of hyperhidrosis. ${ }^{18}$

Pharmacological usage of oxybutynin, an anticholinergic drug, is a valuable option, with specific, positive short-term results in palmar, plantar, axillary and even facial hyperhidrosis. . $119-22^{2}$

Patients with facial hyperhidrosis expect improvements at other hyperhidrosis sites: they wish to stop sweating and carry out their customary daily activities normally. The use of medication takes longer to work (weeks) as opposed to surgery - where results are almost instant. As many patients are eager to achieve good results as quickly as possible, it is important to inform them of the time periods needed for improvement in symptoms. Nevertheless, since pharmacological treatment is systemic, one can expect simultaneous improvement at several hyperhidrosis sites - which is not a feature of the surgical treatment.

Of the 98 initially studied subjects, 7 patients $(7.14 \%)$ failed to return at the $6^{\text {th }}$-week medical appointment. We believe that at least some of these 7 patients, who were lost to follow-up, may have been overly anxious and unwilling to stay on medication that did not provide very short-term results.

Our previous study on facial hyperhidrosis ${ }^{11}$ presented a $24 \%$ follow-up loss (6 patients were lost out of a cohort of 25). We believe that our current (smaller) loss to follow-up may be attributable to our emphatic clarification about pharmacological treatment communicated to patients.

Fourteen patients $(15.38 \%)$ did not have a good initial response to the drug ( 2 had severe dry mouth and 12 did not notice improvement) and thus withdrew from the study.

The remaining $84.61 \%$ of patients $(n=77)$ reported improvement in self-perceived sweating after six weeks of treatment, which was better than previously described $(75 \%)$ in a smaller and shorter study by our group $^{11}$ regarding $\mathrm{FH}$ treatment. In both studies, improvement in QOL was $100 \%$ after six weeks. Specifically in the current study, roughly $60 \%$ reported QOL to be slightly better, whereas the remaining $40 \%$ reported QOL to be much better.

Although an objective assessment of hyperhidrosis is feasible, ${ }^{23}$ we believe that self-reporting is also valuable and appropriate in measuring outcomes in a disease that essentially exhibits symptoms.

Of the 77 patients, as 16 of them had not (yet) completed six months of follow-up, 61 were studied for the long-term results.

Fifty-one $(83.6 \%)$ patients included in the longterm study reported dry mouth, and this complaint as opposed to long-term improvement- was not significantly consistent when we compared patients' perceptions: only $42.7 \%$ of patients remained in the same category (absent/light or moderate/severe) throughout the period studied, $31.1 \%$ improved and 26.2\% reported worsening of this symptom. We could not detect any particular tendency - either improvement or worsening after long-term treatment, since consistency was low (Table 6). Fortunately, no serious side effects (intestinal obstruction, urinary retention) occurred in this series.

In the literature, most studies on the effect of a studied treatment use 2 definite points in time (the first assessment and a fixed, last evaluation date), and their conclusions regarding treatment duration are their main drawback. Patients are usually anxious to know their prognosis and how they will evolve over time. Physicians do not know whether a good outcome after 6 weeks of treatment means that treatment has finished, whether the patient should undergo another type of treatment or continue with the same treatment. This study addresses such questions properly. We focused on patients who had a good initial 
response (six weeks) and who fulfilled our criteria of at least six months of treatment, in order to discover whether the kind of improvement they initially experienced remained in the long-term. We did not exclude patients that were monitored for longer periods: $91.8 \%$ of patients remained in the same improvement category, $3.3 \%$ were "downgraded", and $4.9 \%$ improved; our findings suggest that there is no tachyphylaxis in the use of oxybutynin.

Improvement at other hyperhidrosis sites were only analyzed during the last visit. More than $90 \%$ of patients reported moderate or great improvement at all other hyperhidrosis sites, which is a further benefit of this medication, unlike compensatory hyperhidrosis, which is a side effect of surgical treatment (Table 5).

When a patient decides to undergo surgery for hyperhidrosis, it is essential that they be informed about intra-operative risks (that are fortunately uncommon) and, most importantly, compensatory

\section{REFERENCES}

1. Cerfolio RJ, De Campos JR, Bryant AS, Connery CP, Miller DL, DeCamp MM, et al. The Society of Thoracic Surgeons expert consensus for the surgical treatment of hyperhidrosis. Ann Thorac Surg. 2011;91:1642-8.

2. Strutton DR, Kowalski JW, Glaser DA, Stang PE. US prevalence of hyperhidrosis and impact on individuals with axillary hyperhidrosis: results from a nationalsurvey. J Am Acad Dermatol. 2004;51:241-8.

3. Vorkamp T, Foo FJ, Khan S, Schmitto JD, Wilson P. Hyperhidrosis: evolving concepts and a comprehensive review. Surgeon. 2010;8:287-92.

4. Kao MC, Chen YL, Lin JY, Hsieh CS, Tsai JC. Endoscopic sympathectomy treatment for craniofacial hyperhidrosis. Arch Surg. 1996;131:1091-4.

5. Lin TST, Fang HYH. Transthoracic endoscopic sympathectomy for craniofacial hyperhidrosis: analysis of 46 cases. J Laparoendosc Adv Surg Tech A. 2000;10:243-7.

6. Kim DY, Paik HC, Lee DY. Comparative analysis of T2 selective division of rami-communicantes (ramicotomy) with T2 sympathetic clipping in the treatment of craniofacial hyperhidrosis. Eur J Cardiothorac Surg. 2004;26:396-400.

7. Bell D, Jedynak J, Bell R. Predictors of outcome following endoscopic thoracic sympathectomy. ANZ J Surg. 2014;84:68-72.

8. Try C, Messikh R, Elkhyat A, Aubin F, Humbert RP. Use of oral oxybutynin at $7.5 \mathrm{mg}$ per day in primary hyperhidrosis. Rev Med Liege. 2012;67:520-6.

9. Wolosker N, de Campos JRM, Kauffman P, Puech-Leão P. A randomized placebo-controlled trial of oxybutynin for the initial treatment of palmar and axillary hyperhidrosis. J Vasc Surg. 2012;55:1696-700.

10. Mijnhout GS, Kloosterman H, Simsek S, Strack van Schijndel RJ, Netelenbos JC. Oxybutynin: dry days for patients with hyperhidrosis. Neth J Med. 2006;64:326-8.

11. Wolosker N, Campos JR, Kauffman P, Munia MA, Neves S, Jatene FB, et al. The use of oxybutynin for treating facial hyperhidrosis. An Bras Dermatol. 2011;86:451-6.

12. Campos J, Kauffman P, Werebe EC. Questionnaire of quality of life in patients with primary hyperhidrosis. J Bras Pneumol. 2003;29:178-81.

13. de Campos JR, Kauffman P, Werebe Ede C, Andrade Filho LO, Kusniek S, Wolosker N, et al. Quality of life, before and after thoracic sympathectomy: report on 378 operated patients. Ann Thorac Surg. 2003;76:886-91.

14. Wolosker N, Munia MA, Kauffman P, Campos JR, Yazbek G, Puech-Leão P. Is gender a predictive factor for satisfaction among patients undergoing sympathectomy to treat palmar hyperhidrosis? Clinics (Sao Paulo). 2010;65:583-6.

15. Wolosker N, Krutman M, Campdell TP, Kauffman P, Campos JR, Puech-Leão P. Oxybutynin treatment for hyperhidrosis: a comparative analysis between genders. Einstein (Sao Paulo). 2012;10:405-8. hyperhidrosis (which might affect especially patients who are overweight, as they were in our series). ${ }^{17,24}$

We have shown that anticholinergic treatment is feasible and has good, steady long-term results as regards improvement in excessive sweating, with tolerable side effects. We believe that prior to surgery, every patient should be offered treatment that is reversible before sympathectomy. This approach lowers risks both for patients and physicians, who have occasionally had to cope with patients' regret at the procedure. ${ }^{25}$

\section{CONCLUSION}

Patients treated with oxybutynin for facial hyperhidrosis who experience a good response to treatment in the short-term maintain a good response in long-term treatment. Furthermore, they experience improvement at other hyperhidrosis sites and do not display tachyphilaxis. $\square$

16. Lear W, Kessler E, Solish N, Glaser DA. An epidemiological study of hyperhidrosis. Dermatol Surg. 2007;33:S69-75.

17. de Campos JR, Wolosker N, Takeda FR, Kauffman P, Kuzniec S, Jatene FB, et al. The body mass index and level of resection: predictive factors for compensatory sweating after sympathectomy. Clin Auton Res. 2005;15:116-20.

18. Wolosker N, Krutman M, Kauffman P, Paula RP, Campos JR, Puech-Leão P. Effectiveness of oxybutynin for treatment of hyperhidrosis in overweight and obese patients. Rev Assoc Med Bras. 2013;59:143-7.

19. Tupker RA, Harmsze AM, Deneer VH. Oxybutynin therapy for generalized hyperhidrosis Arch Dermatol. 2006;142:1065-6.

20. Wolosker N, de Campos JR, Kauffman P, Neves S, Yazbek G, Jatene FB, et al. An alternative to treat palmar hyperhidrosis: use of oxybutynin. Clin Auton Res. 2011;21:389-93.

21. Wolosker N, de Campos JR, Kauffman P, Yazbek G, Neves S, Puech-Leao P. Use of oxybutynin for treating plantar hyperhidrosis. Int. J. Dermatol. 2013 May;52(5):620-3.

22. Wolosker N, de Campos JR, Kauffman P, Neves S, Munia MA, Biscegli Jatene F, et al. The Use of Oxybutynin for Treating Axillary Hyperhidrosis. Ann Vasc Surg. 2011;25:1057-62.

23. Ishy A, de Campos JR, Wolosker N, Kauffman P, Tedde ML, Chiavoni CR, et al. Objective evaluation of patients with palmar hyperhidrosis submitted to two levels of sympathectomy: T3 and T4. Interact Cardiovasc Thorac Surg. 2011;12:545-8.

24. de Andrade Filho LO, Kuzniec S, Wolosker N, Yazbek G, Kauffman P, Milanez de Campos JR. Technical difficulties and complications of sympathectomy in the treatment of hyperhidrosis: an analysis of 1731 cases. Ann Vasc Surg. 2013;27:447-53.

25. Smidfelt K, Drott C. Late results of endoscopic thoracic sympathectomy for hyperhidrosis and facial blushing. Br J Surg. 2011;98:1719-24.

\author{
MAILING ADDRESS: \\ Marcelo Passos Teivelis \\ Av. Albert Einstein, 627 - Sala 423 - Bloco A1. \\ 05652-000 - São Paulo - SP \\ Brazil \\ E-mail:dr.marcelo@consultoriovasculares.med.br
}

How to cite this article: Wolosker N, Teivelis MP, Krutman M, Campbell TPDA, Kauffman P, de Campos JR, et al. Long-term results of the use of oxybutynin for the treatment of facial hyperhidrosis. An Bras Dermatol. 2014;89(6):912-6. 